\title{
Optimizing the safety of intradiscal platelet-rich plasma: an in vitro study with Cutibacterium acnes
}

\author{
Meredith H Prysak*,1(D), Cole G Lutz ${ }^{2}$, Tyler A Zukofsky ${ }^{1}$, Jordan M Katz ${ }^{1}$, Peter A Everts ${ }^{3}$ \& \\ Gregory E Lutz ${ }^{2}$ \\ ${ }^{1}$ Orthobond Corporation, 671 Suite B, US 1 North, North Brunswick, NJ 08902, USA \\ ${ }^{2}$ Regenerative SportsCare Institute, 62 East 88th Street, New York, NY 10128, USA \\ ${ }^{3}$ Gulf Coast Biologics, 4331 Veronica S Shoemaker Blvd. \#4 Fort Myers, FL 33916, USA \\ *Author for correspondence: Tel.: +1 732729 6235; Ext.: 14; prysakm@orthobond.com
}

\begin{abstract}
Aim: The most common risk associated with intradiscal injection of platelet-rich plasma (PRP) is discitis with Cutibacterium acnes. It is hypothesized that antimicrobial activity of PRP can be enhanced through inclusion of leukocytes or antibiotics in the injectate. Materials \& methods: Multiple PRP preparations of varying platelet and leukocyte counts were co-cultured with $C$. acnes with or without cefazolin, with viable bacterial colony counts being recovered at 0, 4, 24 and 48 hours post-inoculation. Results: A direct correlation between $C$. acnes recovery and granulocyte counts were observed. Conclusion: We observed the greatest antimicrobial activity with the leukocyte-rich, high platelet PRP preparation combined with an antibiotic in the injectate. However, cefazolin did not completely clear the bacteria in this assay.
\end{abstract}

First draft submitted: 30 July 2019; Accepted for publication: 12 September 2019; Published online: 7 October 2019

Keywords: Cutibacterium acnes $\bullet$ Propionibacterium acnes $\bullet$ anti-infective $\bullet$ antimicrobial $\bullet$ disc degeneration • intervertebral disc $\bullet$ leukocyte $\bullet$ low back pain $\bullet$ neutrophil $\bullet$ platelet-rich plasma $\bullet$ regenerative medicine

There is growing evidence that intradiscal injections of platelet-rich plasma (PRP) can benefit patients with chronic lumbar discogenic pain (reviewed in [1]). Animal studies consistently demonstrate that the injection of PRP locally at the site of damage has the potential to slow the progression of disc degeneration ([2]; reviewed in [3]). These results have also been validated in the clinic, with sustained improvement in function and pain scores sustained for at least 2 years of follow-up [4,5].

There is currently wide variability regarding PRP definitions, preparation methods and resulting PRP bioformulations [6]. Using different preparation protocols during PRP processing, it is possible to yield either a preparation consisting of primarily platelets or a preparation that includes a variety of leukocytes. Furthermore, red blood cell (RBC) concentrations may vary tremendously [7]. Recently, in our practice, we moved to a protocol yielding pure PRP in an effort to maximize platelet concentration. As a byproduct of this new preparation method, granulocytic leukocytes were largely excluded from the preparation. After this change, we encountered three cases of spondylodiscitis postinjection, all of which were culture-confirmed as Cutibacterium (formerly Propionibacterium) acnes (Regenerative Medicine, in press 2019).

C. acnes is a pathogen of increasing interest, particularly for procedures involving the back and shoulders [8]. This gram-positive, aerotolerant, anaerobic commensal organism abundantly colonizes the back and shoulder skin regions which are particularly rich in sebaceous glands, predominantly in men. Due to its slow-growth phenotype and anaerobic growth requirement, it is only recently being appreciated as a clinically relevant pathogen due to improvements in culturing techniques. It is being isolated with increasing frequency during both primary and revision shoulder arthroplasty and instrumented spinal surgery, in numbers approaching $40 \%$ when recovered from explanted hardware [9-13]. Several recent publications have noted the presence of C. acnes in intervertebral disc tissue even before surgery, presumably due to tissue invasion following trauma at the site [14-17]. Our recent clinical experience with spondylodiscitis confirms the published data indicating that infection is a rare but real risk

Future Medicine 
of intradiscal PRP injection, and that $C$. acnes is a major causal organism [18]. Furthermore, it is apparent that $C$. acnes is also an underappreciated pathogen of interest for regenerative medicine techniques involving the spine, such as the intradiscal injection of autologous biologics. Our recent cases of spondylodiscitis after the switch to a leukocyte-poor PRP preparation also raised the question of whether leukocyte exclusion was related to the increasing prevalence of infection we observed, and likewise whether a leukocyte-rich preparation would have a measurable antimicrobial effect against a pathogen such as $C$. acnes in the presence of biologics such as PRP. Defining the effect of platelet concentration and leukocytes on the antimicrobial activity of PRP is therefore of great value and may contribute to minimizing the risk of discitis [19].

In vitro experiments examining this phenomenon have had mixed results, with some experiments showing that leukocytes have antimicrobial activity against gram positive and gram negative organisms, while others lacked efficacy against the same organisms [20-23]. However, there is much heterogeneity in these studies regarding preparation conditions and cellular composition compared with baseline. Additionally, in experiments where the contents of several different preparations were directly compared, the separation between the experimental groups may not be significant enough to correlate positive results with a particular cellular component in the injectate (reviewed in [24]). Furthermore, the inclusion of a prophylactic antibiotic such as cefazolin in the injectate is a common clinical practice but largely unexplored in the literature, particularly as it relates to activity against $C$. acnes. In a study by Intravia et al., cefazolin antimicrobial activity against several strains of bacteria was compared with the antimicrobial activity of PRP with and without leukocytes, but the antibiotic was a separate experimental arm in the study and not examined directly in combination with the PRP as an injectate component [25]. Since disc penetration of certain prophylactic antibiotics can be poor following intravenous administration [26], intradiscal administration of antibiotics may be required for efficacy during this and similar procedures in the clinic.

The purpose of this study is to examine whether the PRP bio-formulation can be optimized to enhance antimicrobial activity against $C$. acnes through both the modulation of platelet concentrations (platelet-rich, PR, $\geq 10 \mathrm{X}$ enrichment; platelet-poor, PP, 3X enrichment), and the inclusion of the leukocyte fraction (particularly granulocyte neutrophils) in the PRP (leukocyte-rich, LR, $\geq 10 \mathrm{X}$; leukocyte-poor, LP, $<1 \mathrm{X}$; leukocyte-ultralow neutrophil, LU, $<0.1 \mathrm{X})$. Additionally, the clinical practice of including a prophylactic dose of cefazolin directly in the PRP injectate has not yet been examined for efficacy against $C$. acnes, and this study will seek to quantify the efficacy of cefazolin inclusion with various PRP formulations. This investigation will be accomplished through a time-kill assay in which PRP preparations of varying platelet and neutrophil concentration are co-cultured with $C$. acnes, and then examined for the recovery of viable bacteria at $0,4,24$ and $48 \mathrm{~h}$ post-inoculation.

\section{Materials \& methods Donors}

Fresh peripheral venous blood was obtained from three healthy volunteers (two male and one female). The following research was reviewed and approved by IntegReview IRB (Austin, TX; OBX-1001), and has been performed in accordance with the ethical standards as laid down in the 1964 Declaration of Helsinki and its later amendments or comparable ethics standards. Written informed consent was obtained from all participants. All donors met the requirements of the American Association of Blood Banks (www.aabb.org) and the American Red Cross (www.redcross.org). There was no selection for age, sex or ethnicity. Donor anonymity was maintained throughout the study.

\section{Experimental design \& study arms}

This study was designed with six different experimental groups: four PRP preparation groups, one whole blood group and one control group containing bacteria in growth media without the addition of human blood product. Each experimental group was further split into two arms: an antibiotic-free arm and its paired arm containing a clinically relevant does of cefazolin. This created 12 different experimental conditions to assess, for a total of 36 samples across the three donors (Table 1). Each sample was assessed for bacterial viability as described below, at four different time points ( $0,4,24$ and $48 \mathrm{~h}$ ). Each donor provided enough peripheral blood to prepare one sample of all experimental blood product groups, to minimize donor-to-donor variability in the experiment.

\section{Preparation of PRP \& whole blood samples}

Fresh blood was obtained from each donor by inserting a $17-\mathrm{G}$ intravenous line into the antecubital vein after the use of sterile preparation. Each donor had $200 \mathrm{ml}$ of peripheral blood drawn for PRP preparation in addition to 


\begin{tabular}{|c|c|c|c|c|}
\hline PRP protocol & Preparation content of PRP & GS60-PurePRP II Kit Yield (ml) & Arm & Cefazolin inclusion $(120 \mu \mathrm{g} / \mathrm{ml})$ \\
\hline$A_{R}$ & $\begin{array}{l}\text { Platelet-rich, leukocyte-poor } \\
\text { (PRPPR LP) } \\
\text { Platelet-poor, } \\
\text { leukocyte-ultralow granulocyte } \\
\text { (PRPPP LU) }\end{array}$ & 3 & $\begin{array}{l}\text { Arm } 1 \\
\text { Arm } 2\end{array}$ & $\begin{array}{l}\text { No } \\
\text { Yes }\end{array}$ \\
\hline$A_{p}$ & & 15 & $\begin{array}{l}\text { Arm } 3 \\
\text { Arm } 4\end{array}$ & $\begin{array}{l}\text { No } \\
\text { Yes }\end{array}$ \\
\hline $\mathrm{B}_{\mathrm{R}}$ & $\begin{array}{l}\text { Platelet-rich, leukocyte-rich } \\
\left(\text { PRP PR LR }^{\text {) }}\right.\end{array}$ & 3 & $\begin{array}{l}\text { Arm } 5 \\
\text { Arm } 6\end{array}$ & $\begin{array}{l}\text { No } \\
\text { Yes }\end{array}$ \\
\hline $\mathrm{B}_{\mathrm{P}}$ & $\begin{array}{l}\text { Platelet-poor, leukocyte-poor } \\
\text { (PRPPP LP) }\end{array}$ & 15 & $\begin{array}{l}\text { Arm } 7 \\
\text { Arm } 8\end{array}$ & $\begin{array}{l}\text { No } \\
\text { Yes }\end{array}$ \\
\hline- & & NA & $\begin{array}{l}\text { Arm } 9 \\
\text { Arm } 10\end{array}$ & $\begin{array}{l}\text { No } \\
\text { Yes }\end{array}$ \\
\hline - & Negative control & NA & Arm 11 & No \\
\hline - & Positive control & NA & Arm 12 & Yes \\
\hline
\end{tabular}

$1 \mathrm{ml}$ of whole blood. Four different PRP preparations and one whole blood control were obtained per donor, for 15 total blood product specimens to be analyzed across all three donors.

All PRP preparations were prepared using the GS60-PurePRP II autologous PRP system, processed by a two-spin procedure with an Executive Series Centrifuge II (EmCyte Corporation; Ft. Myers, FL, USA). Four PRP kits were used per donor. Per kit, $50 \mathrm{ml}$ of peripheral blood was drawn into a $60 \mathrm{cc}$ syringe pre-loaded with $10 \mathrm{ml}$ of sodium citrate and then processed according to one of the four PRP protocols listed in Table $1\left(A_{R}, A_{P}, B_{R}\right.$ and $\left.B_{P}\right)$, according to manufacturer's instructions with minor modifications detailed below.

Protocols $A_{R}$ and $A_{P}$ were run in the following way: $60 \mathrm{ml}$ anticoagulated whole blood was loaded into the concentrating device and centrifuged for $1.5 \mathrm{~min}$ at $3800 \mathrm{RPM}$ (2300 RCF). Platelet plasma suspension (PPS) was then aspirated until RBC filled the aspirating pipe, and the PPS was then transferred into the concentrating accessory and centrifuged for 5 min at 3800 RPM (2300 RCF). Platelet-poor plasma (PPP) was then aspirated off, leaving either 3 or $15 \mathrm{ml}$ (Table 1; Protocols $A_{R}$ or $A_{P}$, respectively) of plasma in the chamber. The platelet buffy coat was then resuspended into the plasma and extracted from the concentrating accessory, yielding the PRP PR LP and PRPP $L$ conditions. Protocols $B_{R}$ and $B_{P}$ were run in a nearly identical manner, except that after the PPS was aspirated, $2 \mathrm{ml}$ of the RBC layer was aspirated from the concentrating device using a separate syringe. The PPS and RBC were then transferred together into the concentrating accessory and centrifuged for 5 min at 3800 RPM (2300 RCF). PPP was then aspirated off, leaving either 3 or $15 \mathrm{ml}$ (Table 1; Protocols $\mathrm{B}_{\mathrm{R}}$ or $\mathrm{B}_{\mathrm{P}}$, respectively) of plasma in the chamber. The platelet buffy coat was then resuspended into the plasma and extracted from the concentrating accessory, yielding the $\mathrm{PRP}_{\mathrm{PR} L \mathrm{R}}$ and $\mathrm{PRP} \mathrm{PP}_{\mathrm{PP}}$ conditions.

\section{Determination of complete blood counts}

Complete blood counts of the 15 specimens of PRP and whole blood were analyzed with a fully automated threepart differential hematology analyzer (ABX Micros ES60; Horiba Ltd., Kyoto, Japan). This was used to determine absolute counts for platelets, RBC, white blood cells (WBC), granulocytes, monocytes and lymphocytes. Note that in this study, the granulocyte fraction is not further subdivided as neutrophil, eosinophil and basophil; however, the granulocyte fraction is understood to be predominantly comprised of neutrophil granulocytes.

\section{Preparation of bacteria \& samples}

A C. acnes phylotype II strain (ATCC 11828) was obtained from the American Type Culture Collection (VA, USA). This strain was subcultured at $37^{\circ} \mathrm{C}$ for $72 \mathrm{~h}$ in Wilkins-Chalgren broth (Oxoid; Hampshire, UK) under anaerobic conditions (GasPak EZ System; BD, NJ, USA) prior to the start of the experiment. To prepare the standardized inoculum for the assay, a $100 \mathrm{ml}$ preparation of $C$. acnes was made by diluting to a density of $1 \times 10^{6} \mathrm{CFU} / \mathrm{ml}$ with fresh Wilkins-Chalgren broth through use of a spectrophotometer (BioMate 3S; ThermoScientific, MA, USA). About $100 \mu \mathrm{l}$ of this standard inoculum was removed and plated according to the description below, to obtain $\mathrm{CFU} / \mathrm{ml}$ counts of the inoculum at the start of the assay. 


\section{Table 2. Average blood cell counts in different whole blood-derived products.}

\begin{tabular}{|c|c|c|c|c|c|c|c|}
\hline PRP Prep & WBC $\left(10^{3} / \mu \mathrm{l}\right)$ & $\operatorname{LYM}\left(10^{3} / \mu \mathrm{I}\right)$ & $\operatorname{MON}\left(10^{3} / \mu \mathrm{I}\right)$ & $\operatorname{GRA}\left(10^{3} / \mu \mathrm{l}\right)$ & $\operatorname{RBC}\left(10^{6} / \mu \mathrm{I}\right)$ & $\operatorname{PLT}\left(10^{3} / \mu \mathrm{I}\right)$ & PRP classification \\
\hline $\mathrm{PRP}_{\mathrm{PR} L \mathrm{P}}$ & $29.30 \pm 1.48$ & $24.20 \pm 1.60$ & $3.00 \pm 0.53$ & $2.10 \pm 0.17$ & $0.27 \pm 0.07$ & $3113 \pm 960$ & PRP IC1 \\
\hline PRP PP LU & $5.80 \pm 0.72$ & $4.87 \pm 0.67$ & $0.47 \pm 0.12$ & $0.47 \pm 0.06$ & $0.06 \pm 0.02$ & $652 \pm 147$ & PRP IA1 \\
\hline 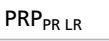 & $40.90 \pm 10.63$ & $24.53 \pm 5.61$ & $4.33 \pm 2.15$ & $12.03 \pm 3.19$ & $2.32 \pm 0.23$ & $2289 \pm 153$ & Red-L-PRP IC1 \\
\hline PRP PP LP $_{1}$ & $9.50 \pm 2.50$ & $6.33 \pm 1.88$ & $0.83 \pm 0.49$ & $2.33 \pm 0.50$ & $0.58 \pm 0.03$ & $674 \pm 152$ & L-PRP IA1 \\
\hline WB & $6.73 \pm 2.66$ & $1.83 \pm 0.32$ & $0.47 \pm 0.38$ & $4.43 \pm 1.99$ & $4.18 \pm 0.73$ & $213 \pm 57$ & - \\
\hline
\end{tabular}

Platelet bioformulation classification indicated.

Errors are standard deviation, $\mathrm{n}=3$.

GRA: Granulocyte; LP: Leukocyte poor; LR: Leukocyte rich; LU: Leukocyte-ultralow granulocyte; LYM: Lymphocyte; MON: Monocytes; PP: Platelet poor; PR: Platelet rich; PRP: Platelet rich plasma; PTL: Platelet; RBC: Red blood cell; WB: Whole blood; WBC: White blood cell.

\section{Co-culturing antimicrobial efficacy assay}

The co-culturing antimicrobial efficacy assay was performed according to the literature with minor modification [22,25]. About $2 \mathrm{ml}$ of the standardized $C$. acnes inoculum was aliquoted per sample well into a sterile, nontissue culture treated 12-well plate (Falcon; NY, USA). For Arms 1 through 10, $0.5 \mathrm{ml}$ of each blood product was added to the well. For Arms 11 through 12, $0.5 \mathrm{ml}$ of 1X PBS (Hyclone; UT, USA) was added as vehicle. For the arms containing antibiotic, cefazolin to a final concentration of $120 \mu \mathrm{g} / \mathrm{ml}$ was added to each sample (Arms 2, 4, 6, 8, 10 and 12). For the antibiotic-free arms (Arms 1, 3, 5, 7, 9 and 11), an equal volume of $1 \mathrm{X}$ PBS was added as vehicle. Multiwell plates were gently swirled to mix the samples.

Samples were incubated on an orbital shaker (MS 3; IKA, Guangzou, China) at $300 \mathrm{rpm}$, at $37^{\circ} \mathrm{C}$ aerobically for up to $48 \mathrm{~h}$. Viable bacteria were recovered from these samples at $0,4,24$ and $48 \mathrm{~h}$ postinoculation with bacteria. At each time point, $100 \mu \mathrm{l}$ was removed from each well. This aliquot was immediately neutralized with Penase Concentrate (BD; NJ, USA) to a final potency of $10,000 \mathrm{LU} / \mathrm{ml} / \mathrm{min}$. This concentration of Penase Concentrate was verified to effectively neutralize the cefazolin concentration used in this assay without compromising bacterial viability (data not shown). A series of tenfold serial dilutions were then prepared. About 3-10 $\mu$ l drops of each dilution were then drop plated according to the Miles and Misra drop plating method onto trypticase soy agar (TSA II) plates with 5\% defibrinated sheep's blood (BD BBL; NJ, USA) [27]. TSA II plates were incubated at $37^{\circ} \mathrm{C}$ for $72 \mathrm{~h}$ under anaerobic conditions, after which colony forming units were determined and recorded. The technicians performing the recovery and plating were blinded to the study groups.

\section{Data analysis}

The $\mathrm{CFU} / \mathrm{ml}$ of recovered $C$. acnes was calculated for all samples. Additionally, percent and $\log _{10}$ reductions for each sample versus negative control and Time 0 values were calculated. Experimental group CBC counts, $\mathrm{CFU} / \mathrm{ml}$ C. acnes counts, and percent efficacy counts were averaged between donors into single group for the purposes of analysis, to minimize donor-to-donor variation. Students' t-tests were performed to assess whether differences in bacterial recovery between experimental groups were significant, $\mathrm{p}$-values $\leq 0.05$ were considered statistically significant.

\section{Results PRP preparations}

Figure 1 and Table 2 summarize the platelet, RBC, granulocyte, monocyte and lymphocyte counts for all products derived from whole blood. The consensus PRP classification from the recently published recommendations from the Platelet Physiology Subcommittee of the Scientific and Standardization Committee have also been included in Table 2 [28]. Both PR products (PRP $P_{\mathrm{PR} L P}, \mathrm{PRP}_{\mathrm{PR} L \mathrm{LR}}$ ) achieved a greater than 10X enrichment of platelets over baseline. Both PP products achieved roughly a $3 \mathrm{X}$ enrichment of platelets. The PRPPP LU product achieved the lowest granulocyte level at $0.47 \pm 0.06\left(10^{3} / \mu \mathrm{l}\right)$ granulocytes, roughly $10 \%$ of the baseline value. This prep had baseline levels of monocytes, and a 2.6X enrichment of lymphocytes. Both the PRP PR LP and PRP PP LP products contained about $50 \%$ less granulocytes than baseline. Lymphocytes exceeded baseline in these preps by $13 \mathrm{X}$ and $3 \mathrm{x}$, respectively. The PRP PR LR product was the only blood product in which average granulocyte counts were substantially enriched on average versus whole blood (12X higher). 


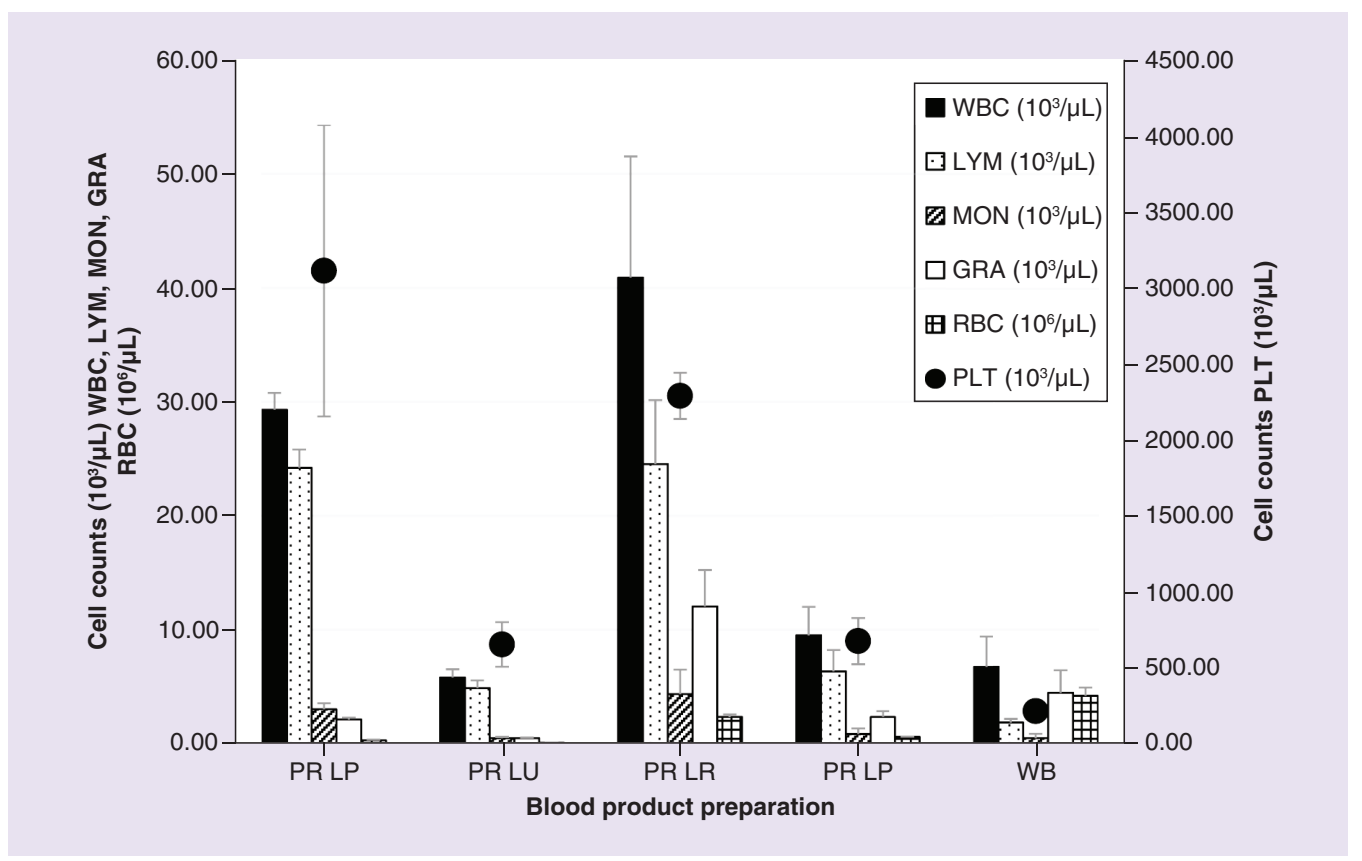

Figure 1. Plot of average blood cell counts in different whole blood-derived products. Black, WBC $\left(10^{3} / \mu \mathrm{l}\right)$; Dotted, $\operatorname{LYM}\left(10^{3} / \mu \mathrm{l}\right)$; Striped, MON $\left(10^{3} / \mu \mathrm{l}\right)$; White, GRA $\left(10^{3} / \mu \mathrm{l}\right)$; Boxed, RBC $\left(10^{6} / \mu \mathrm{l}\right)$; Black circles, PLT $\left(10^{3} / \mu \mathrm{l}\right)$. PLT plotted on secondary $\mathrm{y}$-axis on right. Errors are standard deviation, $\mathrm{n}=3$.

GRA: Granulocyte; LP: Leukocyte-poor; LR: Leukocyte-rich; LU: Leukocyte-ultralow granulocyte; LYM: Lymphocyte; MON: Monocytes; PP: Platelet-poor; PR: Platelet-rich; PRP: Platelet-rich plasma; PLT: Platelet; RBC: Red blood cell; WB: Whole blood; WBC: White blood cell.

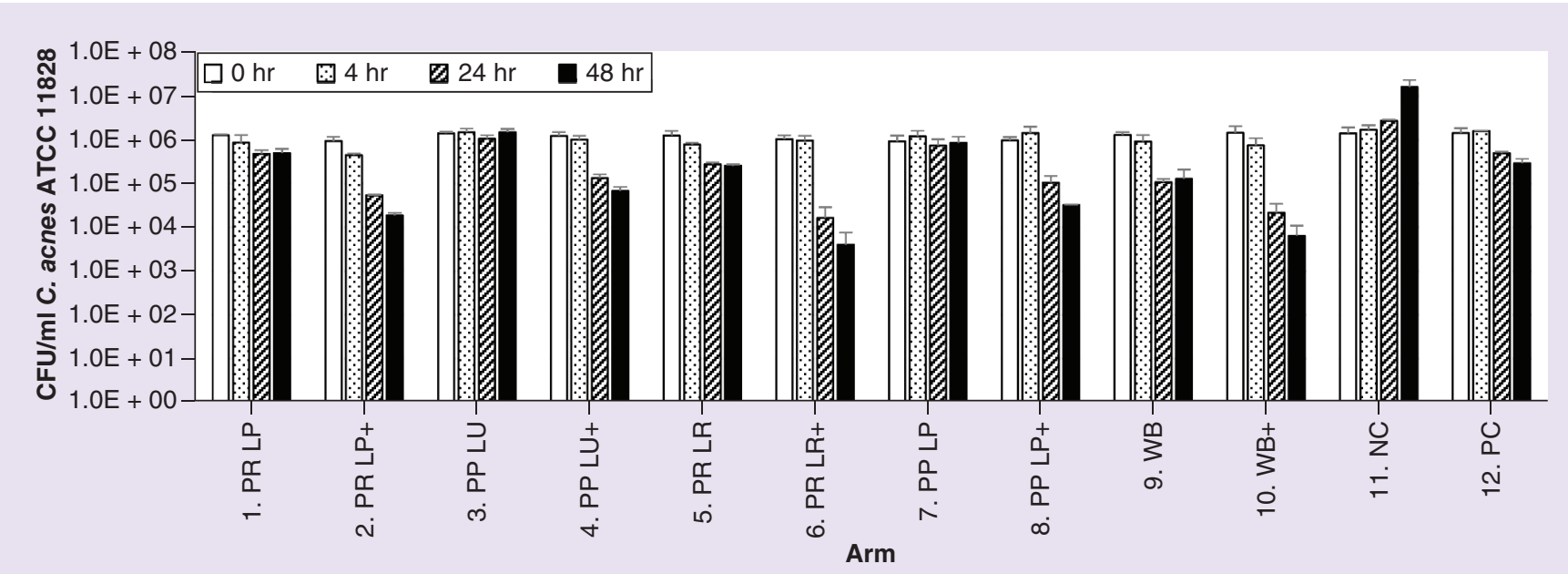

Figure 2. Plot of average CFU/ml of Cutibacterium acnes ATCC 11828 recovered. Time points $0,4,24$ and $48 \mathrm{~h}$ post-exposure. White, $0 \mathrm{~h}$; Dotted, 4 h; Striped, 24 h; Black, 48 h. Errors are standard deviation, $\mathrm{n}=3$.

+: Cefazolin-exposed; LP: Leukocyte poor; LR: Leukocyte rich; LU: Leukocyte-ultralow granulocyte; PR: Platelet rich; PP: Platelet poor; WB: Whole blood.

\section{Co-culturing antimicrobial efficacy assay}

The initial bacterial challenge of $C$. acnes was $1.3 \times 10^{6} \mathrm{CFU} / \mathrm{ml}$ per sample. The average $\mathrm{CFU} / \mathrm{ml}$ recovered per arm at each time point is presented in Figure 2. Arm 11, the negative control $(C$. acnes and media without antibiotics) recovered steadily increasing numbers of $C$. acnes, up to a final total of $1.6 \times 10^{7} \pm 7.2 \times 10^{6} \mathrm{CFU} / \mathrm{ml}$ 
by $48 \mathrm{~h}$. The negative control was the only arm to increase bacterial counts at every time point assayed. In contrast, the addition of cefazolin in the positive control (Arm 12), resulted in a trend of decreasing bacterial counts across all time points, ultimately resulting in a $78 \% \mathrm{CFU} / \mathrm{ml}$ reduction in bacterial counts by $48 \mathrm{~h}$ versus time 0 , and a $98 \% \mathrm{CFU} / \mathrm{ml}$ reduction relative to the negative control.

All experimental arms containing whole blood or whole blood-derived product recovered statistically significantly less $\mathrm{CFU} / \mathrm{ml}$ of $C$. acnes than the negative control at the 24- and 48-h time points. These experimental arms did not increase in bacterial recovery relative to Time 0 . This data demonstrates that all blood product preps (Arms 1 through 10) were capable of controlling the growth of the $C$. acnes contamination. However, when one compares $\mathrm{CFU} / \mathrm{ml}$ counts at later time points to the 0 - $\mathrm{h}$ values for that arm, it is clear that there was no downward trend in bacterial viability for either the PP LU and PP LP conditions in the absence of antibiotics (Arms 3 and 7), with a $\mathrm{CFU} / \mathrm{ml}$ reduction close to zero for the $48 \mathrm{~h}$ time point. The observed antimicrobial activity of these preps can therefore best be described as bacteriostatic. In contrast, a modest but significant downward trend in bacterial viability from time 0 was readily apparent for both the PR LP and PR LR conditions (Arms 1 and 5) even in the absence of antibiotics $(60-80 \% \mathrm{CFU} / \mathrm{ml}$ reduction by $48 \mathrm{~h}$, relative to time 0$)$, demonstrated a mild but marked decrease in bacterial viability over time.

The addition of cefazolin resulted in a more pronounced reduction of bacterial viability over time (1-2 $\log _{10} \mathrm{CFU} / \mathrm{ml}$ reduction) for all antibiotic-containing arms, with the PR LR+ condition (Arm 6) returning the lowest numbers of viable $C$. acnes of any arm Arm 2, 0.005; Arm 4, 0.002; Arm 8, 0.0002; Arm 12, at $4.0 \times 10^{3} \pm 3.6 \times 10^{3} \mathrm{CFU} / \mathrm{ml}$ by $48 \mathrm{~h}$. This represents a $2-\log _{10} \mathrm{CFU} / \mathrm{ml}$ reduction versus time 0 , which is statistically significantly different that either the positive control arm or the other antibiotic-containing arms (p-values: vs Arm 2, 0.005; Arm 4, 0.002; Arm 8, 0.0002; Arm 12, 0.0029). Interestingly, the WB+ condition (Arm 10) returned the second lowest numbers of viable bacteria, for potential reasons that will be further explored in the discussion. It is clear from $C$. acnes $\mathrm{CFU} / \mathrm{ml}$ counts that all of the blood product experimental groups were at a minimum bacteriostatic under assay conditions.

Both PR and LR conditions were associated with the preparations that decreased bacterial viability over time, whereas preparations that were platelet-poor with low leukocyte counts exhibited bacteriostatic behavior without decreasing bacterial viability. While analyzing the data, a potential relationship between absolute leukocyte counts and bacterial recovery was observed. It was noted that the nonantibiotic and antibiotic arm with the lowest average $\mathrm{CFU} / \mathrm{ml}$ recovery of bacteria at 24 and $48 \mathrm{~h}$ (Arms 5 PR LR and Arm 6 PR LR+, respectively) were made from the whole blood-derived product $\left(\mathrm{PRP}_{\mathrm{PR} \mathrm{LR}}\right.$ ) with the highest leukocyte counts.

Interestingly, the nonantibiotic arm and antibiotic arm with the second lowest average recovery of bacteria at 24 and 48 h (Arm 9 WB and Arm $10 \mathrm{WB}+$, respectively) were whole blood, which had the second-lowest leukocyte counts of any blood-product but had the second highest granulocyte counts. To understand more about the relationship between $C$. acnes $\mathrm{CFU} / \mathrm{ml}$ recovery and the different leukocyte components of the blood-derived products, the recovered $\mathrm{CFU} / \mathrm{ml}$ for each donor was converted to $\log _{10}$ reduction versus its respective Time $0 \mathrm{CFU} / \mathrm{ml}$ counts, and plotted versus the granulocyte counts for each donor (Figure 3). This was done separately for the antibiotic-free arms (Figure 3A; Arms 1, 3, 5, 7 and 9) and cefazolin arms (Figure 3B; Arms 2, 4, 6, 8 and 10). Arms 11 and 12 were not included in the analysis since they did not contain blood product. The equation of a best fit linear trendline was then calculated for each plot along with the coefficient of determination $\left(\mathrm{R}^{2}\right)$. The results, while not perfectly linear, show a clear positive correlation between $\log$ reduction of $C$. acnes and granulocyte counts, with a positive slope and $\mathrm{R}^{2}$ value of 0.64 .

This plot was repeated for both the monocyte counts (Figure 4) and the lymphocyte counts (Figure 5). For both the preparations without (Figure 4A) and with (Figure 4B) cefazolin, there was little apparent relationship between monocyte counts and $\log _{10}$ reduction of $C$. acnes $\mathrm{CFU} / \mathrm{ml}$, with an $\mathrm{R}^{2}$ value less than 0.3 in both cases. Likewise, there was no relationship detected between lymphocyte counts and $\log _{10}$ reduction of $C$. acnes $C F U / \mathrm{ml}$ without (Figure 5A) and with (Figure 5B) cefazolin. Since both platelet-rich conditions evidenced a drop in $C$. acnes $\mathrm{CFU} / \mathrm{ml}$ recovery over time, individual platelet counts per donor without (Figure $6 \mathrm{~A}$ ) and with cefazolin (Figure 6B) were also plotted with the C. acnes $\log _{10}$ reduction versus Time 0 . With an $\mathrm{R}^{2}$ value approaching zero, it is clear that a relationship does not exist between absolute platelet counts and a drop in $C$. acnes viability as measured by $\mathrm{CFU} / \mathrm{ml}$ reduction over time under the conditions of this assay. 

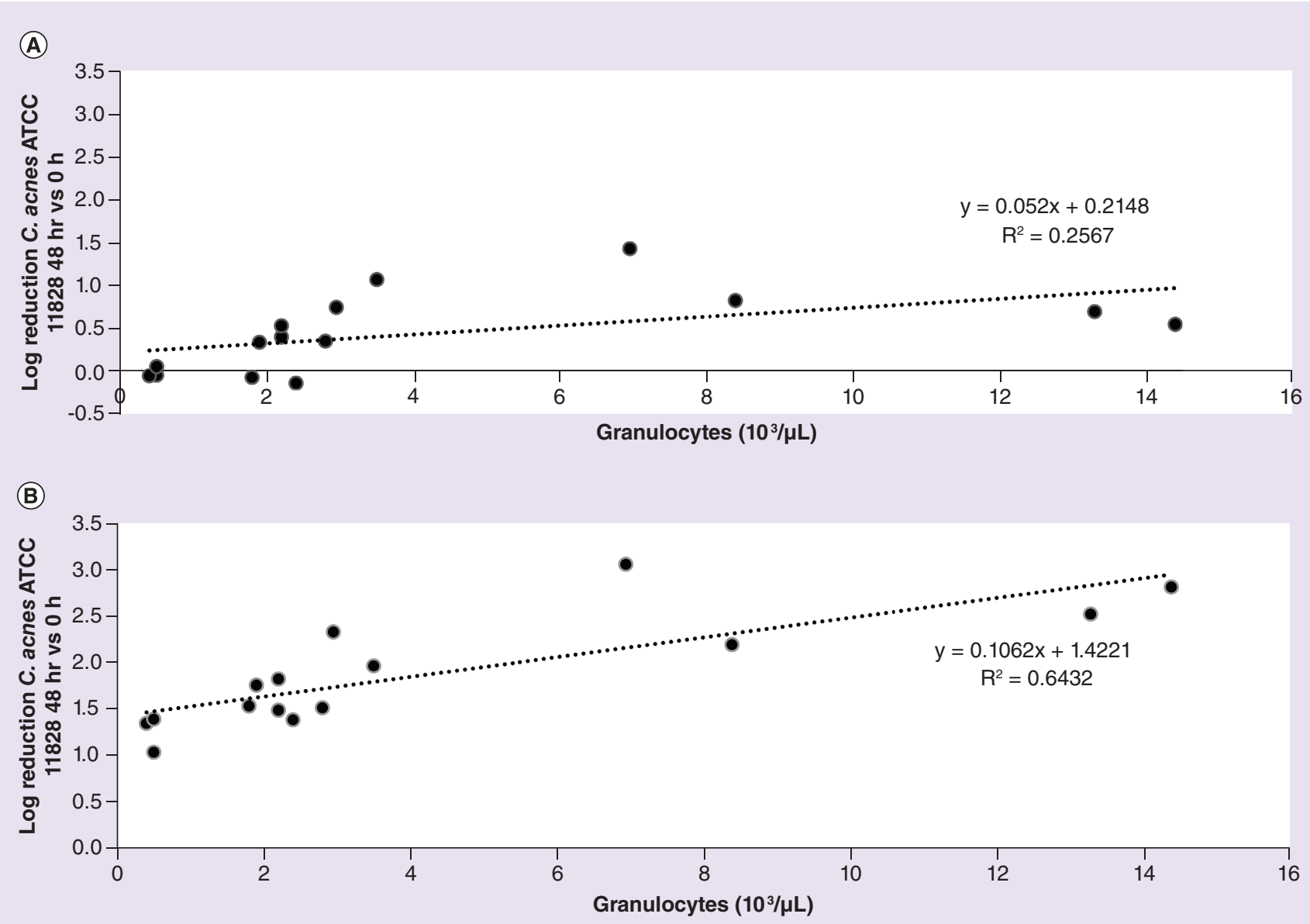

Figure 3. $\mathrm{CFU} / \mathrm{ml} \log _{10}$ reduction versus granulocyte counts per donor. $\log _{10}$ reductions at $48 \mathrm{~h}$ calculated versus average Time $0 \mathrm{CFU} / \mathrm{ml}$ recovery. (A) Data for antibiotic-free Arms 1, 3, 5, 7 and 9. (B) Data for cefazolin Arms 2, 4, 6, 8 and 10.

\section{Discussion}

To our knowledge, this study is one of the first to examine the direct effect of varying PRP cellular components on $C$. acnes. The significant stratification between blood cell counts for the different preparations allowed for the detailed analysis of sufficiently distinct PRP conditions, facilitating the identification of antimicrobial trends correlated to absolute blood cell count.

This study also evaluated the addition of antibiotics with the varying PRP bioformulations against $C$. acnes, a pathogen of increasing significance. Interestingly, blood-derived products, regardless of content, displayed at least a minimal inherent bacteriostatic activity under the conditions of this assay, although in some cases this was clearly due to bacteriostatic and not bactericidal action. The addition of cefazolin to the blood product resulted in an additional $1 \log _{10}$ of $\mathrm{CFU} / \mathrm{ml}$ reduction minimum by $48 \mathrm{~h}$ versus the nonantibiotic comparator from the same condition for every noantibiotic/cefazolin pair of samples. The magnitude of the additive effect of cefazolin was consistent with the activity of the positive control (Arm 12, cefazolin without blood product), which also resulted a nearly $1 \log _{10}$ reduction of $C$. acnes $\mathrm{CFU} / \mathrm{ml}$ versus the initial inoculum. The antimicrobial activity of the sodium citrate used as an anticoagulant during this study was evaluated in an independent investigation (data not shown), and it was found that sodium citrate was mildly antimicrobial, and the final sodium citrate concentration in this assay is likely sufficient to exert the bacteriostatic effect observed for the platelet-poor samples, although it cannot account for the drop in bacterial viability observed for bioformulations containing platelet and/or leukocyte-rich conditions. However, since the amount and choice of compound used as an anticoagulant varies among PRP isolate kits, this variable may be another opportunity to optimize PRP injectate to enhance patient outcomes while limiting risks. 

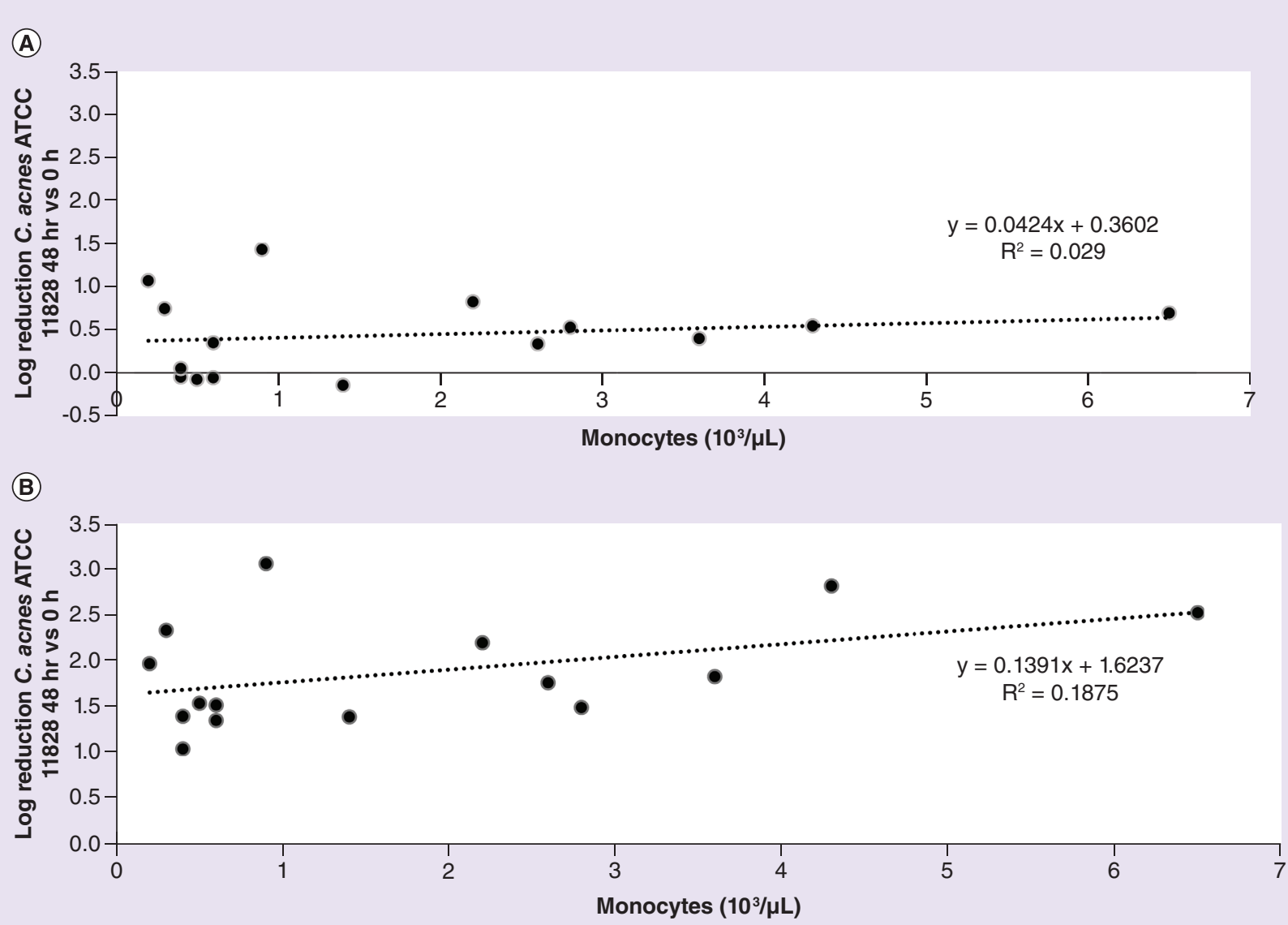

Figure 4. $\mathrm{CFU} / \mathrm{ml} \log _{10}$ reduction versus monocyte counts per donor. $\log _{10}$ reductions at $48 \mathrm{~h}$ calculated versus time $0 \mathrm{CFU} / \mathrm{ml}$ recovery. (A) Data for antibiotic-free Arms 1, 3, 5, 7 and 9. (B) Data for cefazolin Arms 2, 4, 6, 8 and 10.

Intriguingly, there was a clear relationship between the numbers of recovered $C$. acnes over time versus the absolute granulocyte counts, evident when comparing the average recovered $C$. acnes $\mathrm{CFU} / \mathrm{ml}$ counts for the PR LP+ and PR LR+ conditions (Arms 2 and 6, respectively). The CFU/ml reduction at $48 \mathrm{~h}$ was statistically significantly different between these two conditions ( $p$-value $=0.004$; Student's t-test, Type 2). Even more intriguing, the unexpectedly efficacious activity of the whole blood fractions (Arms 9 and 10) was consistent with the absolute counts of granulocytes in those samples, which were the second highest counts of all study arms. Analysis of individual donor data demonstrated that granulocyte counts are positively correlated with the observed decrease in bacterial viability observed over time in this assay. Trends were not observed between viability and monocyte, lymphocyte or platelet counts.

Interestingly, although a relationship exists between granulocyte counts and bacterial recovery, the dynamics are likely more complex. Arms 1 and 7 (PR LP and PP LP, respectively) had similar granulocyte counts, but Arm 1 (PR LP) demonstrated a drop in bacterial viability over time and Arm 3 (PP LP) did not. Therefore, granulocyte counts alone cannot account for the drop in bacterial viability over time. The PR LP condition had over four-times the platelet count of the PP LP condition, and it would be tempting to postulate that platelets or platelet-released factors may contribute to antimicrobial activity of blood product in a way that is not linked to absolute cell counts. However, the WB condition (Arm 9) has 3X less platelets than either leukocyte-poor condition of Arms 3 and 7 while still evidencing a downward trend in viability over time; thus, it is possible that an additional component is involved in the switch between bacteriostatic and bactericidal activity of the blood-derived products under the conditions of this assay in a way that is not directly coupled to absolute platelet counts. 

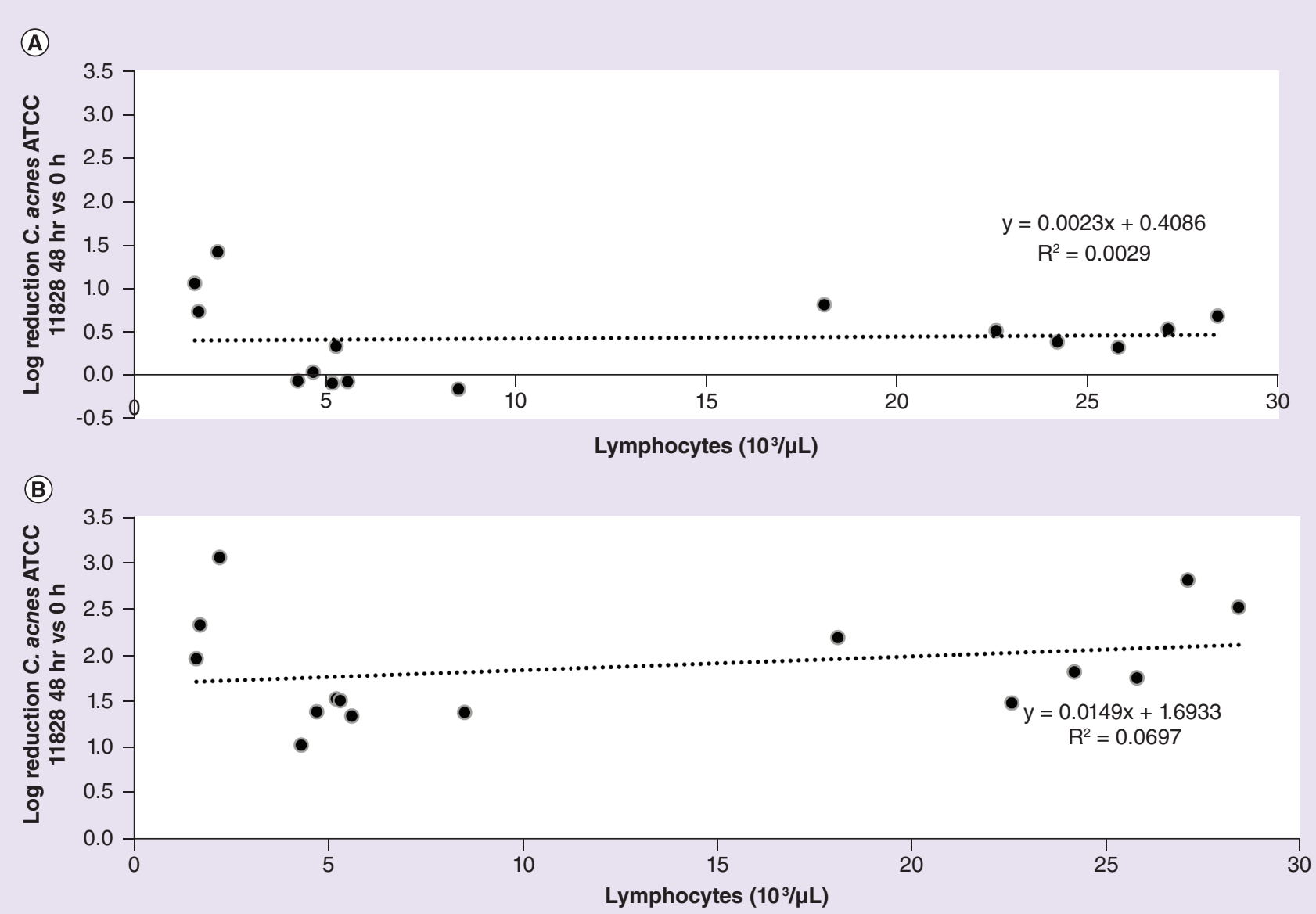

Figure 5. CFU/ml $\log _{10}$ reduction versus lymphocyte counts per donor. $\log _{10}$ reductions at $48 \mathrm{~h}$ calculated versus average time $0 \mathrm{CFU} / \mathrm{ml}$ recovery. (A) Data for antibiotic-free Arms 1, 3, 5, 7 and 9. (B) Data for cefazolin Arms 2, 4, 6, 8 and 10.

\section{Conclusion}

There is a clear effect on antibacterial activity against $C$. acnes in this study when leukocytes and/or cefazolin are included as PRP components. A direct relationship between absolute cell count and decreases in bacterial viability were observed for granulocyte counts, but not for monocyte, lymphocyte or platelet counts. Cefazolin addition to $120 \mu \mathrm{g} / \mathrm{ml}$ resulted in a modest decrease in bacterial viability, but was insufficient to address the full bioburden present in this study.

\section{Translational perspective}

This study has several important implications that require further study. The first is that a PRP bioformulation containing higher levels of granulocytes may have an advantage over a PRP bioformulation containing low levels of granulocytes with regard to impeding $C$. acnes growth at the injection site. However, this advantage would have to be weighed against patient outcomes using injectate with higher levels of leukocytes. The literature is inconsistent on whether the presence of (granulocytic/monocytic) leukocytes are beneficial for tissue repair. For muscle and tendon repair, there are studies suggesting that a full buffy coat leukocyte-rich PRP promotes an inflammatory response [29-31]; conversely, there are also studies showing that leukocyte-rich PRP are effective in the treatment of tendinopathy [32,33]. Likewise, there are mixed results for the treatment of knee joint cartilage degeneration showing both positive [34,35] and negative [36,37] effects of granulocytic leukocyte-rich PRP on healing. Interestingly, there is also evidence that the presence of leukocytes enhance platelet functioning in PRP [38]. It would seem based on the literature that the indication of use and preparation manner are crucial. To date clinical data have demonstrated efficacy with leukocyte-poor PRP in treating degenerative disc disease but has not directly compared the efficacy of leukocyte-rich versus leukocyte-poor PRP preparations, thus it is not known if one preparation has superior 

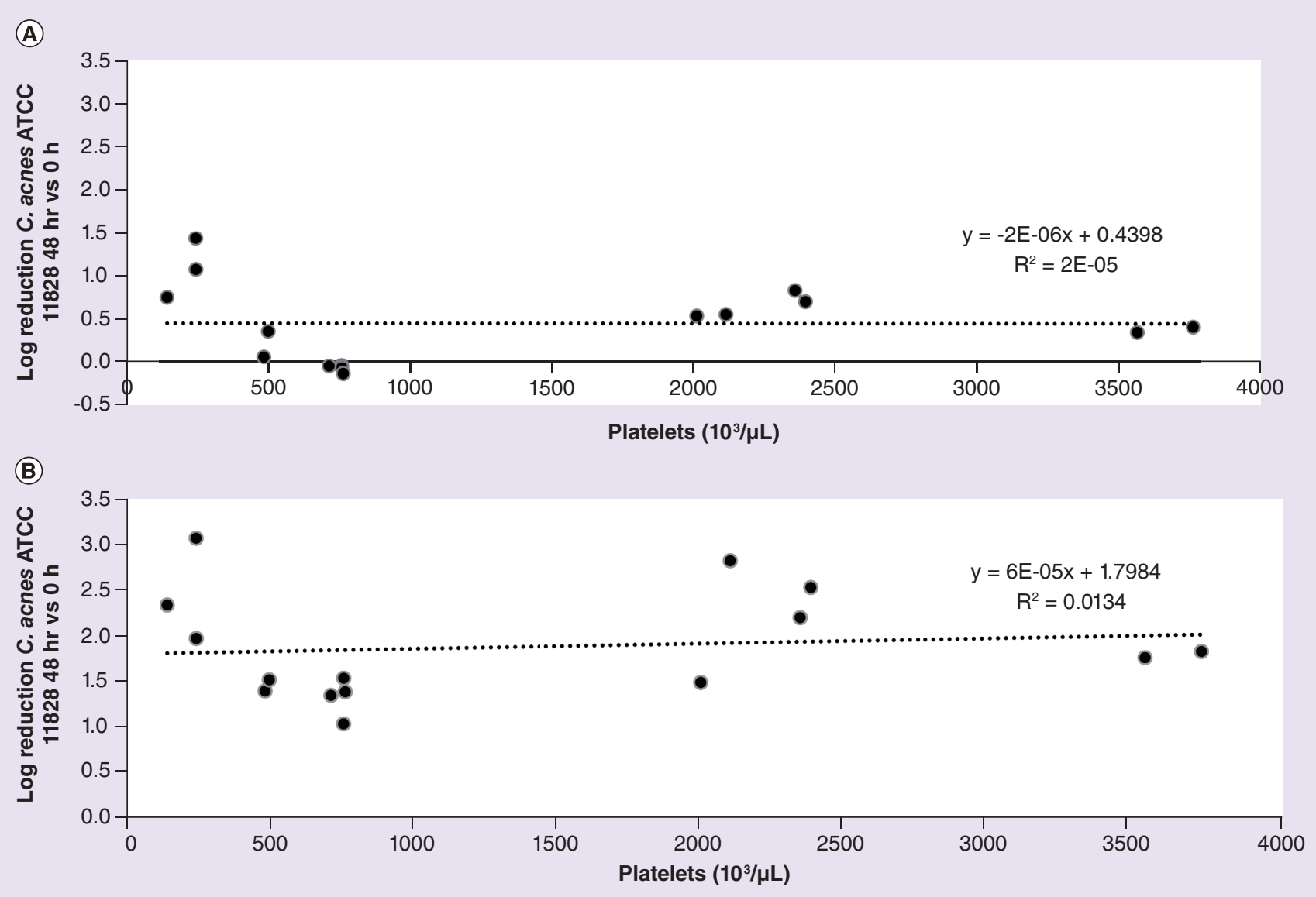

Figure 6. $\mathrm{CFU} / \mathrm{ml} \log _{10}$ reduction versus platelet counts per donor. $\log _{10}$ reductions at $48 \mathrm{~h}$ calculated versus average Time $0 \mathrm{CFU} / \mathrm{ml}$ recovery. (A) Data for antibiotic-free Arms 1, 3, 5, 7 and 9. (B) Data for cefazolin Arms 2, 4, 6, 8 and 10.

activity to the other. There may be an opportunity, therefore, to mitigate the risk of infection without impacting outcomes through a minor modulation of the PRP content toward a higher granulocyte condition. The data in this paper suggests, however, that there might be a critical threshold of granulocytes required to see this antibacterial effect, which requires further characterization. It will also be of future interest to examine the findings of this study in the context of platelet-rich fibrin, which presents platelets and high levels of leukocytes in a fibrin matrix in the absence of anticoagulants.

Second, the use of an antibiotic added to the injectate resulted in a decrease in bacterial recovery. The addition of antibiotics to PRP has not yet been correlated to patient outcomes in the literature. Analysis of platelet functioning when PRP and antibiotics are combined together in the injectate will be the subject of future studies in order to further characterize the effects of such clinical practices.

At last, the amount of cefazolin currently used in the clinic $(120-500 \mu \mathrm{g} / \mathrm{ml})$ was not sufficient to eradicate the full inoculum of $C$. acnes under the conditions of this study, even though the study was performed under aerobic conditions, when $C$. acnes would be expected to be most vulnerable to the antibiotic as suggested by susceptibility data gathered in a separate study (data not shown). Furthermore, the prolonged ( $48 \mathrm{~h}$ ) contact at the full concentration of cefazolin in this study may not translate to the clinical situation, where the antibiotic may dissipate over time, thereby lowering the local concentration. The data gathered during this study, therefore, represents a best-case scenario for addressing $C$. acnes contamination at the injection site. This study suggests that other antibiotics should be tested for potential utility in the clinic for the goal of specifically mitigating the risk of C. acnes contamination during PRP injection.

It is perhaps not surprising that cefazolin inadequately addresses $C$. acnes contamination, since this practice was adopted before it was fully understood that $C$. acnes contamination was so pervasive during shoulder and back 
procedures. The increase in the isolation of $C$. acnes in the clinic has been largely attributed to improved detection techniques, including anaerobic culturing and longer incubation times. It is also possible that the use of prophylactic treatments to which it is not susceptible facilitates the identification of $C$. acnes contamination once the competing (and often faster growing) flora have been eliminated.

Given recent data suggesting that $C$. acnes contamination is more prevalent than previously thought, coupled with new data suggesting that $C$. acnes biofilms may already be present in damaged IVDs, designing infection-mitigation strategies that include $C$. acnes as a likely pathogen of interest may help mitigate the risk of infection associated with PRP injections in the future.

\section{Limitation}

There are several limitations to this study. First, the in vitro conditions cannot perfectly model the in vivo microenvironment that the $C$. acnes and PRP are exposed to, where antibiotic and bacterial concentrations would be in constant flux. Although the difference between bacteriostatic and bactericidal activity against a particular organism is pronounced in vitro, the clinical efficacy does not always mirror this behavior [39]. Second, although granulocyte levels were able to be modulated in relation to platelet levels, a condition that was platelet-poor and leukocyte-rich was not tested due to limitations in the methods used to prepare the PRP. Therefore, granulocyte activity at baseline or sub-baseline levels of platelets will be the subject of future investigations. Additionally, growth factor analysis was not performed in this study in order to examine the effects of the cefazolin dose on the growth factor activity in the PRP preparations, due to limited sample volumes. Therefore, it is not known what effect including antibiotics in the injectate will have on the activity of the cells in the local microenvironment. Future studies are also planned to investigate the effects of varying PRP bioformulations on $C$. acnes cultured under anaerobic conditions to better mimic the intradiscal environment.

\section{Summary points}

- Cutibacterium acnes has recently been identified as a pathogenic organism of interest, particularly in procedures involving the shoulders and back, and leads to poor patient outcomes.

- Infection, particularly with C. acnes is a potential risk during intradiscal injection procedures, such as with platelet-rich plasma (PRP).

- Examining whether the antimicrobial activity of PRP can be enhanced through the inclusion of leukocytes (particularly neutrophils) in the preparation, or the inclusion of prophylactic antibiotics directly in the injectate may help control this risk and lead to better patient outcomes.

- PRP of varying platelet and neutrophil content were co-cultured with $\mathrm{C}$. acnes with or without cefazolin for up to $48 \mathrm{~h}$.

- All blood products controlled the growth of $C$. acnes in a way that cannot be fully explained by the antimicrobial properties of the anticoagulant.

- The presence of neutrophils resulted in a time-dependent decrease in C. acnes recovery that was statistically significantly less than conditions without high levels of neutrophils.

- Cefazolin imparted an additive antimicrobial effect but did not clear all of the $C$. acnes under these conditions.

- The absolute granulocyte and monocyte counts were clearly correlated with the $\log _{10}$ reduction of $C$. acnes.

- The inclusion of neutrophils and a prophylactic antibiotic impart enhanced antimicrobial activity to the PRP.

- Because cefazolin showed limited activity against $C$. acnes, other prophylactic antibiotics should be explored to address this pathogen.

- Future studies should define the effects of prophylactic antibiotics in the injectate on the activity of the platelets through analysis of growth factors and other parameters.

\section{Author contributions}

MH Prysak focused on concept and design of study, execution of study, data acquisition, analysis and interpretation, manuscript drafting and critical revising. PA Everts contributed to the design of study, data interpretation and critical revising of the manuscript. JM Katz was involved in data interpretation and critical review of the manuscript. CG Lutz and TA Zukofsky carried out data acquisition and analysis of data, and revising manuscript. GE Lutz contributed to concept and design of study, acquisition of data, data interpretation, and critical revision of manuscript. 


\section{Acknowledgments}

The authors gratefully acknowledge Ewa Profiruk of EmCyte Corporation for her help in preparing the PRP bioformulations, and Dr Nick Beatty, DO for critical reading of this manuscript.

\section{Financial \& competing interests disclosure}

The authors gratefully acknowledge the Regenerative SportsCare Foundation and EmCyte Corporation for financial support of this study. G Lutz acted as the study doctor of the following research with human participants in conjunction with Orthobond Corporation, and serves as CEO of Orthobond Corporation; he is also the founder of the Regenerative SportsCare Foundation. The terms of this arrangement have been reviewed and approved by IntegReview IRB (Austin, TX; OBX-1001) in accordance with its policy on objectivity in research. P Everts serves as the Chief Scientific Officer of EmCyte Corporation. The authors have no other relevant affiliations or financial involvement with any organization or entity with a financial interest in or financial conflict with the subject matter or materials discussed in the manuscript apart from those disclosed.

No writing assistance was utilized in the production of this manuscript.

\section{Ethical conduct of research}

The authors state that they have obtained appropriate institutional review board approval for this study (IntegReview IRB, Austin, TX; OBX-1001). In addition, written informed consent was obtained from all study participants.

\section{Open access}

This work is licensed under the Attribution-NonCommercial-NoDerivatives 4.0 Unported License. To view a copy of this license, visit http://creativecommons.org/licenses/by-nc-nd/4.0/

\section{References}

1. Akeda K, Yamada J, Linn ET, Sudo A, Masuda K. Platelet-rich plasma in the management of chronic low back pain: a critical review. $J$. Pain Res. 12, 753-767 (2019).

2. Obata $\mathrm{S}$, Akeda $\mathrm{K}$, Imanishi $\mathrm{T}$ et al. Effect of autologous platelet-rich plasma-releasate on intervertebral disc degeneration in the rabbit anular puncture model: a preclinical study. Arthritis Res. Ther. 14(6), R241 (2012).

3. Li P, Zhang R, Zhou Q. Efficacy of platelet-rich plasma in retarding intervertebral disc degeneration: a meta-analysis of animal studies. Biomed. Res. Int. 7919201 (2017).

4. Monfett M, Harrison J, Boachie-Adjei K, Lutz G. Intradiscal platelet-rich plasma (PRP) injections for discogenic low back pain: an update. Int. Orthop. 40(6), 1321-1328 (2016).

5. Tuakli-Wosornu YA, Terry A, Boachie-Adjei K et al. Lumbar intradiskal platelet-rich plasma (PRP) injections: a prospective, double-blind, randomized controlled study. PM R 8(1), 1-10 (2016).

6. Mazzocca AD, Mccarthy MB, Chowaniec DM et al. Platelet-rich plasma differs according to preparation method and human variability. J. Bone Joint Surg. Am. 94(4), 308-316 (2012).

7. Everts PA. Autologous platelet-rich plasma and mesenchymal stem cells for the treatment of chronic wounds. In: KamilHakan Dogan (Ed.) Wound Healing: Current Perspectives, IntechOpen (2019). https://www.intechopen.com/books/wound-healing-current-perspectives

8. Achermann Y, Goldstein EJ, Coenye T, Shirtliff ME. Propionibacterium acnes: from commensal to opportunistic biofilm-associated implant pathogen. Clin. Microbiol. Rev. 27(3), 419-440 (2014).

9. Falconer TM, Baba M, Kruse LM et al. Contamination of the surgical field with propionibacterium acnes in primary shoulder arthroplasty. J. Bone Joint Surg. Am. 98(20), 1722-1728 (2016).

10. Grosso MJ, Sabesan VJ, Ho JC, Ricchetti ET, Iannotti JP. Reinfection rates after 1-stage revision shoulder arthroplasty for patients with unexpected positive intraoperative cultures. J. Shoulder Elbow Surg. 21(6), 754-758 (2012).

11. Sampedro MF, Huddleston PM, Piper KE et al. A biofilm approach to detect bacteria on removed spinal implants. Spine 35(12), 1218-1224 (2010).

12. Torrens C, Mari R, Alier A, Puig L, Santana F, Corvec S. Cutibacterium acnes in primary reverse shoulder arthroplasty: from skin to deep layers. J. Shoulder Elbow Surg. 28(5), 839-846 (2019).

13. Wang B, Toye B, Desjardins M, Lapner P, Lee C. A 7-year retrospective review from 2005 to 2011 of Propionibacterium acnes shoulder infections in Ottawa, Ontario, Canada. Diagn. Microbiol. Infect. Dis. 75(2), 195-199 (2013).

14. Agarwal V, Golish S, Alamin T. Bacteriologic culture of excised intervertebral disc from mmunocompetent patients undergoing single level primary lumbar microdiscectomy. J. Spinal Disord. Tech. 24(6), 397-400 (2011).

15. Capoor MN, Ruzicka F, Machackova T et al. Prevalence of propionibacterium acnes in intervertebral discs of patients undergoing lumbar microdiscectomy: a prospective cross-sectional study. PLoS ONE 11(8), e0161676 (2016). 
16. Capoor MN, Ruzicka F, Schmitz JE et al. Propionibacterium acnes biofilm is present in intervertebral discs of patients undergoing microdiscectomy. PLoS ONE 12(4), e0174518 (2017).

17. Chen Y, Wang X, Zhang X et al. Low virulence bacterial infections in cervical intervertebral discs: a prospective case series. Eur. Spine J. 27(10), 2496-2505 (2018).

18. Pobiel RS, Schellhas KP, Pollei SR, Johnson BA, Golden MJ, Eklund JA. Diskography: infectious complications from a series of 12,634 cases. AJNR Am. J. Neuroradiol. 27(9), 1930-1932 (2006).

19. Tang YQ, Yeaman MR, Selsted ME. Antimicrobial peptides from human platelets. Infect. Immun. 70(12), 6524-6533 (2002).

20. Bielecki T, Gazdzik T, Arendt J, Szczepanski T, Krol W, Wielkoszynski T. Antibacterial effect of autologous platelet gel enriched with growth factors and other active substances. J. Bone Joint Surg. Br. 89(3), 417-420 (2007).

21. Burnouf T, Chou ML, Wu YW, Su CY, Lee LW. Antimicrobial activity of platelet (PLT)-poor plasma, PLT-rich plasma, PLT gel, and solvent/detergent-treated PLT lysate biomaterials against wound bacteria. Transfusion 53(1), 138-146 (2013).

22. Moojen DJ, Everts PA, Schure RM et al. Antimicrobial activity of platelet-leukocyte gel against Staphylococcus aureus. J. Orthop. Res. 26(3), 404-410 (2008).

23. Tohidnezhad M, Varoga D, Wruck CJ et al. Platelets display potent antimicrobial activity and release human beta-defensin 2. Platelets 23(3), 217-223 (2012).

24. D'asta F, Halstead F, Harrison P, Zecchi Orlandini S, Moiemen N, Lord J. The contribution of leucocytes to the antimicrobial activity of platelet-rich plasma preparations: a systematic review. Platelets 29(1), 9-20 (2018).

25. Intravia J, Allen $\mathrm{D}$, Durant $\mathrm{T}$ et al. In vitro evaluation of the anti-bacterial effect of two preparations of platelet rich plasma compared with cefazolin and whole blood. Muscles Liaments Tendons J. 4(1), 79-84 (2014).

26. Capoor MN, Lochman J, Mcdowell A et al. Intervertebral disc penetration by antibiotics used prophylactically in spinal surgery: implications for the current standards and treatment of disc infections. Eur. Spine J. 28(4), 783-791 (2019).

27. Miles AA, Misra SS. The estimation of the bactericidal power of the blood. J. Hyg. (London) 38(6), 732-749 (1938).

28. Harrison P. Subcommittee on Platelet P. The use of platelets in regenerative medicine and proposal for a new classification system: guidance from the SSC of the ISTH. J. Thromb. Haemost. 16(9), 1895-1900 (2018).

29. Dragoo JL, Braun HJ, Durham JL et al. Comparison of the acute inflammatory response of two commercial platelet-rich plasma systems in healthy rabbit tendons. Am. J. Sports Med. 40(6), 1274-1281 (2012).

30. Mccarrel TM, Minas T, Fortier LA. Optimization of leukocyte concentration in platelet-rich plasma for the treatment of tendinopathy. J. Bone Joint Surg. Am. 94(19), e143(1-8) (2012).

31. Tidball JG. Inflammatory processes in muscle injury and repair. Am. J. Physiol. Regul. Integr. Comp. Physiol. 288(2), R345-R353 (2005).

32. Fitzpatrick J, Bulsara M, Zheng MH. The effectiveness of platelet-rich plasma in the treatment of tendinopathy: a meta-analysis of randomized controlled clinical trials. Am. J. Sports Med. 45(1), 226-233 (2017).

33. Fitzpatrick J, Bulsara MK, O'donnell J, Mccrory PR, Zheng MH. The effectiveness of platelet-rich plasma injections in gluteal tendinopathy: a randomized, double-blind controlled trial comparing a single platelet-rich plasma injection with a single corticosteroid injection. Am. J. Sports Med. 46(4), 933-939 (2018).

34. Mariani E, Canella V, Cattini L et al. Leukocyte-rich platelet-rich plasma injections do not up-modulate intra-articular pro-inflammatory cytokines in the osteoarthritic knee. PLoS ONE 11(6), e0156137 (2016).

35. Riboh JC, Saltzman BM, Yanke AB, Fortier L, Cole BJ. Effect of leukocyte concentration on the efficacy of platelet-rich plasma in the treatment of knee osteoarthritis. Am. J. Sports Med. 44(3), 792-800 (2016).

36. Filardo G, Kon E, Pereira Ruiz MT et al. Platelet-rich plasma intra-articular injections for cartilage degeneration and osteoarthritis: single-versus double-spinning approach. Knee Surg. Sports Traumatol. Arthrosc. 20(10), 2082-2091 (2012).

37. Milants C, Bruyere O, Kaux JF. Responders to platelet-rich plasma in osteoarthritis: a technical analysis. Biomed. Res. Int. 2017, 7538604 (2017).

38. Parrish WR, Roides B, Hwang J, Mafilios M, Story B, Bhattacharyya S. Normal platelet function in platelet concentrates requires non-platelet cells: a comparative in vitro evaluation of leucocyte-rich (type 1a) and leucocyte-poor (type 3b) platelet concentrates. BMJ Open Sport Exerc. Med. 2(1), e000071 (2016).

39. Pankey GA, Sabath LD. Clinical relevance of bacteriostatic versus bactericidal mechanisms of action in the treatment of gram-positive bacterial infections. Clin. Infect. Dis. 38, 864-870 (2004). 